\title{
Crystalline silica exposure, radiological silicosis, and lung cancer mortality in diatomaceous earth industry workers
}

\author{
Harvey Checkoway, Janet M Hughes, Hans Weill, Noah S Seixas, Paul A Demers
}

Department of Environmental Health H Checkoway N S Seixas

Department of Epidemiology H Checkoway

Tulane University, New Orleans, Louisiana, USA

Department of Biostatistics and Epidemiology J W Hughes $\mathrm{H}$ Weill

Department of Medicine $\mathrm{H}$ Weill

Occupational Hygiene Programme, University of British Columbia, Vancouver, British Columbia, Canada

P A Demers

Correspondence to: Dr H Checkoway, University of Washington, Department of Environmental Health, Box 357234, Seattle, Washington 98195-7234, USA.

Received 6 May 1998 Returned to author 10 July 1998

Revised manuscript received 20 August 1998 Accepted for publication 8 September 1998

\begin{abstract}
Background-The role of silicosis as either a necessary or incidental condition in silica associated lung cancer remains unresolved. To address this issue a cohort analysis of dose-response relations for crystalline silica and lung cancer mortality was conducted among diatomaceous earth workers classified according to the presence or absence of radiological silicosis.
\end{abstract}

Methods-Radiological silicosis was determined by median 1980 International Labour Organisation system readings of a panel of three " $B$ " readers for 1809 of 2342 white male workers in a diatomaceous earth facility in California. Standardised mortality ratios (SMR) for lung cancer, based on United States rates for 1942-94, were calculated separately for workers with and without radiological silicosis according to cumulative exposures to respirable crystalline silica (milligrams per cubic meter $\times$ years; $\mathbf{m g} / \mathrm{m}^{3}$-years) lagged 15 years.

Results-Eighty one cases of silicosis were identified, including 77 with small opacities of $\geqslant 1 / 0$ and four with large opacities. A slightly larger excess of lung cancer was found among the subjects with silicosis (SMR 1.57, 95\% confidence interval (CI) 0.43 to 4.03 ) than in workers without silicosis (SMR 1.19, 95\% CI 0.87 to 1.57). An association between silica exposure and lung cancer risk was detected among those without silicosis; a statistically significant $(p=0.02)$ increasing trend of lung cancer risk was seen with cumulative exposure, with SMR reaching 2.40 (95\% CI 1.24 to 4.20 ) at the highest exposure level $\left(\geqslant 5.0 \mathrm{mg} / \mathrm{m}^{3}\right.$-years). A similar statistically significant $(\mathrm{p}=0.02)$ dose-response gradient was observed among non-silicotic subjects when follow up was truncated at 15 years after the final negative radiograph (SMR 2.96, 95\% CI 1.19 to 6.08 at $\geqslant 5.0 \mathrm{mg} / \mathrm{m}^{3}$-years), indicating that the association among non-silicotic subjects was unlikely to be accounted for by undetected radiological silicosis.

Conclusions-The dose-response relation observed between cumulative exposure to respirable crystalline silica and lung cancer mortality among workers without radiological silicosis suggests that silicosis is not a necessary co-condition for silica related lung carcinogenesis. However, the relatively small number of silicosis cases in the cohort and the absence of radiographic data after employment limit interpretations.

(Thorax 1999;54:56-59)

Keywords: silicosis, silica dust, lung cancer

The International Agency for Research on Cancer (IARC) recently concluded that there is sufficient evidence that inhaled crystalline silica is a human lung carcinogen. ${ }^{1}$ Pronounced increases in the risk of lung cancer have been documented consistently among cohorts of registered or hospitalised silicotic subjects, ${ }^{2}$ and data from these studies were regarded as supportive evidence by the IARC. ${ }^{1}$ However, the potential for selection and confounding biases in epidemiological studies of registered silicotic subjects has been noted. ${ }^{3}$ In a recent review of the literature on crystalline silica and lung cancer Weill and $\mathrm{McDonald}^{4}$ reported that, among defined occupational cohorts, there was little evidence of excess risk among workers without silicosis. One interpretation of this observation is that the increase in lung cancer limited to subjects with silicosis is consistent with a dose-response relation with crystalline silica, assuming that silicotic subjects typically have the highest exposures. Alternatively, pulmonary fibrosis may play a necessary contributory role in lung carcinogenesis, which would imply that those with silicosis represent a susceptible subgroup. The latter explanation finds support from investigations showing that other types of lung fibrosis are associated with excess lung cancer. In studies of workers in the manufacture of asbestos products ${ }^{5}$ and asbestos miners, ${ }^{6}$ excess lung cancer was detected only among those with radiographic ${ }^{5}$ or pathological ${ }^{6}$ asbestosis. Furthermore, exceptionally high risks of lung cancer have been observed in patients with idiopathic pulmonary fibrosis. ${ }^{7}$ In any case, clarification of the relation between silicosis and lung cancer risk would improve the understanding of the underlying biological mechanisms of lung carcinogenicity and would have important implications for establishing acceptable occupational exposure levels.

Data from a cohort study of mortality and radiographically identified silicosis among diatomaceous earth workers ${ }^{89}$ provided a valuable opportunity to examine the association between crystalline silica exposures, pulmonary fibrosis, and lung cancer. A particular advantage of our study is the availability of both quantitative exposure estimates and historical 
radiographs for a large segment of the cohort that permitted determinations of the temporal relations between exposure, the onset of radiographic abnormalities, and risk of lung cancer. Our objective was to estimate dose-response relations of crystalline silica and lung cancer among workers with and without radiographic evidence of silicosis. These analyses were intended to address the issue of the necessity of silicosis for an increased silica related risk of lung cancer.

\section{Methods}

STUDY SETTING AND POPULATION

Details of the original cohort mortality and silicosis studies have been described previously $^{89}$ and will only be summarised briefly here. The study was conducted among workers from one diatomaceous earth mining and processing facility in Lompoc, California. Diatomaceous earth, which is derived from skeletal remains of diatoms, has been mined at the plant since 1908. Calcining of the ore produces a final product consisting of $10-60 \%$ crystalline silica, mainly in the form of cristobalite. The cohort included 2342 white men employed for at least 12 months cumulative service and at least one day between 1 January 1942 and 31 December 1987. Mortality follow up of the cohort was performed from 1 January 1942 to 31 December 1994, during which time 77 deaths from lung cancer were identified.

RADIOGRAPHIC DETERMINATION OF SILICOSIS There were 1809 men $(77 \%)$ for whom readable chest radiographs were available from the company's radiographic surveillance programme that has been in effect since the 1930s. This number excludes 169 workers whose only radiographs were taken at the time of hire or within the first month of employment; three of these workers, one of whom died from lung cancer, had initial radiographs consistent with pneumoconiosis. ${ }^{9}$ Final radiographs of acceptable quality were read by a panel of three experienced " $B$ " readers according to the 1980 International Labour Organisation (ILO) system $^{10}$ to identify the prevalence of pneumoconiosis, defined as either median ILO profusion category $1 / 0$ and higher or the presence of large opacities. Dates of silicosis onset for the cases were estimated as the median dates of the initial films with ILO category $1 / 0$ or higher or large opacity, based on three readers' reviews of earlier radiographs. There were 81 cases of

Table 1 Lung cancer mortality by ILO radiographic category and cumulative exposure to crystalline silica

\begin{tabular}{|c|c|c|c|c|c|c|}
\hline \multirow{3}{*}{$\begin{array}{l}\text { Cumulative } \\
\text { exposure } \\
\text { (mg/m } / \mathrm{m}^{3} \text {-year) }\end{array}$} & \multicolumn{6}{|c|}{ Radiographic category } \\
\hline & \multicolumn{3}{|c|}{$<1 / 0(n=1798) *$} & \multicolumn{3}{|c|}{$\geqslant 1 / 0$ or large opacity $(n=81)$} \\
\hline & Deaths & $S M R$ & $(95 \% C I)$ & Deaths & $S M R$ & $(95 \% C I)$ \\
\hline$<0.5$ & 13 & 1.05 & $(0.56$ to 1.79$)$ & 0 & 0 & (0 to 12.2 ) \\
\hline $0.5-1.9$ & 13 & 0.86 & (0.46 to 1.48$)$ & 0 & 0 & (0 to 19.3 ) \\
\hline $2.0-4.9$ & 10 & 1.25 & (0.60 to 2.29$)$ & 0 & 0 & (0 to 5.32 ) \\
\hline$\geqslant 5.0$ & 12 & 2.40 & ( 1.24 to 4.20$)$ & 4 & 2.94 & (0.80 to 7.53$)$ \\
\hline Total & 48 & 1.19 & (0.87 to 1.57$)$ & 4 & 1.57 & (0.43 to 4.03$)$ \\
\hline
\end{tabular}

*Includes 1728 with $<1 / 0$ throughout, and 70 who later converted to $\geqslant 1 / 0$

SMR = standardised mortality ratio based on rates for US white men, 1942-94.

$95 \% \mathrm{CI}=95 \%$ confidence interval for SMR pneumoconiosis, 77 with small opacity profusion scores of $1 / 0$ and higher and four with large opacities. The mean age at onset was 42 years (range 26-65). Among the 77 cases of silicosis with small opacities there were 62 $(81 \%)$ with median ILO profusion scores of $1 / 0$ to $1 / 2$, eight $(10 \%)$ with category $2 / 1$, and seven $(9 \%)$ with category $2 / 2$ and higher. ${ }^{9}$

ESTIMATION OF CRYSTALLINE SILICA EXPOSURE The cumulative exposures of members of the cohort to respirable crystalline silica were estimated from historical occupational hygiene data linked to detailed employment records. ${ }^{8}$ Linear regression modelling was applied to estimate job and time period specific exposure intensities in units of milligrams per cubic meter $\left(\mathrm{mg} / \mathrm{m}^{3}\right)$, taking into account temporal differences in occupational hygiene techniques including the change from impinger to gravimetric dust measurement methods. ${ }^{11}$

\section{STATISTICAL ANALYSIS}

Observed lung cancer mortality among workers, classified according to the presence or absence of radiological silicosis, was compared with expected values based on rates for white men in the USA during 1942-97. These comparisons were expressed as standardised mortality ratios (SMRs) adjusted for age and calendar year. For workers identified as cases of radiological silicosis, person-years of observation were assigned to the non-silicosis category until the dates of onset of silicosis to maintain the appropriate time dependency in the analysis. There were 70 cases of silicosis in whom onset of the disease occurred after the start of follow up (1942), who thus contributed person-years to both the non-silicosis and silicosis groups; the remaining 11 cases of silicosis were diagnosed before 1942 and contributed all person-years to the silicosis group. As in the earlier report, ${ }^{8}$ exposures were lagged by 15 years to allow for disease latency. Trends of SMRs by cumulative exposure were tested for statistical significance, assuming that observed/ expected ratios followed an exponential function with cumulative exposure. ${ }^{12}$

\section{Results}

As shown in table 1, the lung cancer excess compared with national rates was slightly larger among workers with radiological silicosis (SMR $1.57,95 \%$ CI 0.43 to 4.03 ) than in workers without silicosis (SMR 1.19, 95\% CI 0.87 to 1.57); however, the excess in those with silicosis was based on only four deaths, all at the highest exposure level ( $\geqslant 5.0 \mathrm{mg} / \mathrm{m}^{3}$-years). There was a statistically significant $(\mathrm{p}=0.02)$ positive gradient of increasing risk with cumulative exposures among workers without radiological silicosis; the SMR reached 2.40 (95\% CI 1.24 to 4.20) in the highest exposure stratum. The lung cancer excess among the 81 cases of silicosis was confined to workers with the highest exposures (SMR 2.94, 95\% CI 0.80 to 7.53 ).

We considered the possibility that the observed exposure-response trends of crystalline silica and lung cancer were confounded by cigarette smoking by examining the relation 
between smoking prevalence and exposure. Smoking data were available for $58 \%$ of workers included in these analyses, and were only adequate to classify workers as ever smokers or never smokers. The percentages of ever smokers among non-silicotic workers with available smoking data for the four exposure categories of crystalline silica were, respectively, $63.5 \%$, $80.5 \%, 85.1 \%$, and $78.9 \%$. We applied Axelson's method ${ }^{13}$ of indirect adjustment for confounding, assuming a 20 -fold increased risk of lung cancer for smokers compared with nonsmokers. This distribution of smoking prevalence in those without silicosis would result in a relative risk of 1.22 due to confounding for the comparison of the highest with the lowest exposure stratum. The observed ratio of SMRs in table 1 for these exposure strata is 2.29 $(2.40 / 1.05)$, which would be reduced to 1.88 based on the estimated extent of confounding. The corresponding percentages of ever smokers, by exposure category, among the 81 cases of silicosis were $66.7 \%, 100 \%, 100 \%$, and $94.7 \%$. Adjustment of relative risks for differences in smoking prevalence is complicated for those with silicosis because all four lung cancer deaths occurred among workers in the highest exposure stratum. However, if it is assumed that the SMR for the lowest exposure category is 1.05 (as was seen for non-silicotic subjects) then, following the same approach as for nonsilicotics, the smoking adjusted relative risk for lung cancer between the highest and lowest exposure categories in subjects with silicosis would be 2.04

Radiographic surveillance of the cohort did not extend beyond dates of employment termination. Thus, there is the possibility that some workers whose final radiographs were read as $<1 / 0$ may have ultimately developed radiologi$\mathrm{cal}$ silicosis that was not detected. Evidence that this may have occurred is indicated by the difference in mean duration of time from first hire to final radiograph between workers classified as having silicosis (21.3 years) and those whose final radiographs were negative (11.5 years). The corresponding mean ages at final radiograph were 51.9 and 37.7 years for silicotic and non-silicotic subjects, respectively. In order to evaluate possible bias due to such misclassification we computed SMRs by exposure category among non-silicotic subjects with follow up truncated at 15 years after the final negative radiograph. Fifteen years was chosen as the cut off for follow up because, although some additional cases of radiological silicosis

Table 2 Lung cancer mortality by cumulative exposure to crystalline silica among workers with ILO radiographic category $<1 / 0$, first 15 years of follow up after final negative radiograph

\begin{tabular}{llll}
\hline $\begin{array}{l}\text { Cumulative exposure } \\
\left(m g / m^{3} \text {-year }\right)\end{array}$ & Deaths & SMR & (95\% CI) \\
\hline$<0.5$ & 6 & 1.04 & $(0.38$ to 2.27$)$ \\
$0.5-1.9$ & 3 & 0.79 & $(0.16$ to 2.31$)$ \\
$2.0-4.9$ & 6 & 1.81 & $(0.66$ to 3.93$)$ \\
$\geqslant 5.0$ & 7 & 2.96 & $(1.19$ to 6.08$)$ \\
Total & 22 & 1.44 & $(0.90$ to 2.19$)$ \\
\hline
\end{tabular}

SMR = standardised mortality ratio based on rates for US white men, 1942-94.

$95 \% \mathrm{CI}=95 \%$ confidence interval for SMR. may have occurred in this period, the study that linked lung fibrosis with radiological asbestosis ${ }^{5}$ demonstrated a role for lung fibrosis with a comparable follow up interval. Also, 15 years provided a sufficiently long period to observe subsequent lung cancer mortality. Although based on smaller numbers, the SMR trend with cumulative exposure (table 2 ) remained statistically significant $(p=0.02)$ and was not materially different from that observed in full follow up of non-silicotic subjects (table 1).

\section{Discussion}

Our findings indicate that the increased risk of lung cancer related to cumulative exposure to respirable crystalline silica in this cohort of diatomaceous earth workers was not restricted to workers with radiological silicosis. Information on cigarette smoking, a potentially important confounding factor, was limited, which precluded a full assessment of possible bias. Nonetheless, the available smoking data suggested that confounding was unlikely to be the main explanation for the findings. A notable strength of our study was the availability of both detailed exposure data that enabled a quantitative dose-response estimation and serial radiographs for a large fraction of the cohort $(77 \%)$ that were read to identify dates of onset of silicosis. Nearly all other studies that have addressed the relation between silicosis and lung cancer have relied on radiographic or pathological data obtained at a single point in time, either during or after employment. However, the limited statistical precision of our data, due to the relatively small number of workers with silicosis $(n=81)$ and lung cancer deaths among that group $(n=4)$, should be recognised.

The results from this group of diatomaceous earth workers are at variance with findings from studies of US metal miners, ${ }^{14}$ Dutch ceramics workers, ${ }^{15}$ and Australian gold miners ${ }^{16}$ in which excess mortality from lung cancer was concentrated in subjects with silicosis, identified either radiographically ${ }^{14}{ }^{15}$ or from medical compensation records. ${ }^{16}$ However, exposure assessment for crystalline silica in these other studies was based on relatively crude dose indicators such as years of underground mining ${ }^{14}$ or ordinal rankings of jobs, ${ }^{15}{ }^{16}$ thus hindering comparisons with results from our study which benefited from exposure quantitation. Another perhaps more direct evaluation of the role of silicosis in lung cancer risk is provided by a case-control study nested within a cohort of South African gold miners. ${ }^{17}$ That study, which examined the joint effects of quantitative estimates of crystalline silica exposure and pathologically confirmed silicosis (determined at necropsy) on lung cancer mortality, revealed a dose-response gradient with cumulative exposure and an apparently stronger independent effect of silicosis. However, the authors ${ }^{17}$ could not reach a firm conclusion as to whether silicosis is a necessary co-condition for silica associated lung carcinogenesis or an unrelated consequence of exposure. Our findings are in qualitative agreement with the study of South African gold miners with regard to a dose-response gradient for 
crystalline silica that is not restricted to cases of silicosis. It should also be noted that comparisons across studies may be complicated by differences in exposure types; in our study cristobalite was the main form of crystalline silica while in other settings quartz is predominant. Differences in carcinogenic potential among the various crystalline silica polymorphs have not yet been established. ${ }^{1}$

Interpretation of our results and of similar investigations should be tempered by the realisation of the inherent complexities of epidemiological investigations of the interrelations between silica, silicosis, and lung cancer. Chief among these is the predictably strong correlation between silica exposure and silicosis which complicates attempts to disentangle unique contributions to the aetiology of lung cancer. This was noted in the study of South African gold miners ${ }^{17}$ and is certainly true in our cohort among whom a very strong dose-response relation between cumulative exposure to crystalline silica and radiological silicosis has been demonstrated previously. ${ }^{9}$ Furthermore, radiographic surveillance of worker populations typically ends when workers leave employment, which can result in underestimates of the true prevalence of silicosis. Such underestimates could have resulted in misclassification of silicosis that would bias the assessment of the role of silicosis in the excess risk of lung cancer. Our study may have suffered from this problem, as have others. ${ }^{14-16}$ There are also some well known limitations of chest radiographs, especially their low sensitivity to detect microscopic fibrotic nodules. ${ }^{18}$ Pathological detection of silicosis at necropsy, as was performed in the South African gold miner study, ${ }^{17}$ offers a more accurate and valid method of silicosis classification than radiographs. However, for epidemiological purposes this advantage may be offset by feasibility constraints and the absence of information on the times of onset of silicosis that can be inferred from serial chest radiographs.

An understanding of the nature and extent of the contribution of pulmonary fibrosis to the pathogenesis of lung cancer in populations exposed to silica would undoubtedly advance our knowledge of the mechanisms of occupational lung carcinogenesis. To date, the epidemiological findings bearing on this issue, including data from our study, are conflicting. The role of silicosis in lung cancer is likely to remain controversial until large scale epide- miological studies that incorporate valid and sensitive longitudinal measurements of both silica induced lung fibrosis and quantified exposures to silica are conducted. Obtaining data on potentially important confounders, especially cigarette smoking, will also be necessary. In the interim, appropriately designed toxicological experiments may provide the clearest insights into the underlying biology of the association between silica, silicosis, and lung cancer.

This project was supported by grant R01 OH03126 from the US National Institute for Occupational Safety and Health. The authors are grateful to Drs Brian Boehlecke, Anthony Proto, and Jerome Wiot for reading chest radiographs.

1 International Agency for Research on Cancer: Evaluation of Carcinogenic Risks to Humans. Silica, some silicates, coal dust, and para-aramid fibrils. Volume 68. Lyon, France: IARC Publications, 1997.

2 Smith AH, Lopipero PA, Barroga VR. Meta-analysis of studies of lung cancer among silicotics. Epidemiology 1995; 6:617-24

$3 \mathrm{McDonald}$ C. Silica, silicosis, and lung cancer; an epidemiological update. Appl Occup Environ Hyg 1995;10: 1056-63.

4 Weill $\mathrm{H}, \mathrm{McD}$ onald JC. Exposure to crystalline silica and risk of lung cancer: the epidemiological evidence. Thorax 1996;51:97-102

5 Hughes JM, Weill $\mathrm{H}$. Asbestosis as a precursor of asbestos related lung cancer: results of a prospective mortality study. related lung cancer: results of a pro
Br F Ind Med 1991;48:229-33.

6 Sluis-Cremer G, Bezuidenhout BN. Relation between Sluis-Cremer G, Bezuidenhout BN. Relation between
asbestosis and bronchial cancer in amphibole asbestos miners. Br f Ind Med 1989;46:537-40.

7 Turner-Warwick M, Lebowitz M, Burrows B, et al. Cryptogenic fibrosing alveolitis and lung cancer. Thorax 1980;35:496-9

8 Checkoway H, Heyer NJ, Seixas NS, et al. Dose-response associations of silica with nonmalignant respiratory disease and lung cancer mortality in the diatomaceous earth industry. Am 7 Epidemiol 1997;145:680-8.

9 Hughes JM, Weill H, Checkoway H, et al. Radiographic evidence of silicosis risk in the diatomaceous earth industry. Am 7 Resp Crit Care Med 1998;158:807-14.

10 International Labour Office. Guidelines for the use of the ILO international classification of radiographs for pneumoconiosis. No. 22 (rev). Geneva: World Health Organization, Occupational Safety and Health Services, 1980.

11 Seixas NS, Heyer NJ, Welp E, et al. Quantitation of historical exposures in the diatomaceous earth industry. Ann Occup Hyg 1997;41:591-604

12 Breslow NE, Day NE. Statistical methods in cancer research. Vol. II. The analysis of cohort studies. Lyon, France: IARC Publications, 1987

13 Axelson $\mathrm{O}$, Steenland $\mathrm{K}$. Indirect methods of assessing the effects of tobacco use in occupational studies. $A m \mathcal{F}$ Ind Med 1988;13:105-18.

14 Amandus H, Costello J. Silicosis and lung cancer in US metal miners. Arch Environ Health 1991;46:82-9.

15 Meijers JMM, Swean GMH, Slangen JJM. Mortality and lung cancer in ceramic workers in the Netherlands: preliminary results. Am f Ind Med 1996;30:26-30.

16 de Klerk NH, Musk AW. Silica, compensated silicosis, and lung cancer in Western Australian goldminers. Occup Environ Med 1998;55:243-8.

17 Hnizdo E, Murray J, Klempman S. Lung cancer in relation to exposure to silica dust, silicosis, and uranium production in South African gold miners. Thorax 1997;52:271-5.

18 Hnizdo E, Murray J, Sluis-Cremer GK, et al. Correlation between radiological and pathological diagnosis of silicosis: an autopsy population based study. Am F Ind Med 1993;24: $427-45$. 